\title{
High-contrast top-emitting organic light-emitting devices for active-matrix displays
}

\author{
Chih-Jen Yang, Chun-Liang Lin, and Chung-Chih $\mathrm{Wu}^{\mathrm{a})}$ \\ Department of Electrical Engineering, Graduate Institute of Electro-optical Engineering, and Graduate \\ Institute of Electronics Engineering, National Taiwan University, Taipei, Taiwan 10617, \\ Republic of China \\ Yung-Hui Yeh, ${ }^{\text {a) }}$ Chun-Cheng Cheng, Yen-Hsun Kuo, and Tai-Hong Chen \\ Electronics Research and Service Organization (ERSO), Industrial Technology Research Institute (ITRI), \\ Hsin-Chu, Taiwan 300, Republic of China
}

(Received 21 April 2005; accepted 11 August 2005; published online 29 September 2005)

\begin{abstract}
Unlike previous high-contrast devices that all involve inserting extra layer(s) with optical purposes (e.g., absorption and interference) into the active region of devices, in this-letter we report a high-contrast top-emitting organic light-emitting device (OLED) that utilizes only optical characteristics of electrodes and anti-reflection coatings deposited outside the active region, thus reducing the complexity of devices. Furthermore, the device has an inherent microcavity which is beneficial to electroluminescence efficiency. The devices are readily compatible with the processing of active-matrix backplanes, and active-matrix OLED displays incorporating such high-contrast top-emitting devices were demonstrated to have improved readability under a strong lighting environment. (c) 2005 American Institute of Physics. [DOI: 10.1063/1.2081137]
\end{abstract}

Organic light-emitting devices (OLEDs), either conventional bottom emitting or top emitting, in general are composed of a reflective back electrode, organic layers, and a semitransparent exit electrode for light out-coupling. ${ }^{1,2}$ With the reflective back electrode, OLEDs in general exhibit rather strong reflection. Such reflection would degrade the contrast of an OLED display under strong ambient illumination. Polarizer or filter films may be laminated on the surface of the display panel to reduce reflection of ambient illumination. ${ }^{3}$ However, the use of such contrastenhancement films reduces the power efficiency of the displays below $\sim 40 \%$ of the device efficiency, and also adds extra complexity and cost in fabrication. OLED structures that could integrate the characteristics of low ambient-light reflection within the OLED structure and yet retain as much emission efficiency as possible will thus be particularly useful, since the display contrast could then be enhanced with reduced impact on cost and efficiency.

The few reported high-contrast OLED structures ${ }^{3-6}$ all deal with bottom-emitting OLEDs and may not be readily adapted for top-emitting OLEDs, which have a few technical merits (e.g., larger aperture ratios) over bottom-emitting devices for high-performance active-matrix OLED displays (AMOLEDs). Furthermore, these previous structures all involve inserting extra layer(s) with optical purposes (e.g., absorption, interference, etc.) into the active region of devices (i.e., between the anode and the cathode). Thus one must be concerned about both optical and electrical characteristics (i.e., carrier injection and transport) of such layers. In this letter, we report a high-contrast OLED structure that utilizes only optical characteristics of electrodes and anti-reflection (AR) coatings deposited outside the active region of devices, thus reducing the impact on electrical characteristics and device complexity. Such a structure is implemented into top-

\footnotetext{
a) Authors to whom correspondence should be addressed; electronic mail:
} chungwu@cc.ee.ntu.edu.tw, yhyeh@itri.org.tw emitting OLEDs, with the results of improved efficiency compared to that of conventional OLEDs laminated with polarizers, and compatibility with active-matrix backplanes. AMOLEDs incorporating such high-contrast top-emitting OLEDs and thus possessing improved readability under a strong lighting environment are also demonstrated.

The structure of the top-emitting OLED investigated is shown in Fig. 1(a), which uses a reflective metal as the bottom anode and a thin semitransparent metal film as the top cathode. Mo, with a high work function and a moderate reflectivity $(\sim 50 \%-60 \%)$, is used as the bottom anode for hole injection and as one mirror in the structure. The organic multilayer structure in sequence consists of $4,4^{\prime}, 4^{\prime \prime}$-tris(3-methylphenylphenylamino)triphenylamine (m-MTDATA, $30 \mathrm{~nm}$ ) as the hole-injection layer, $\alpha$-naphthylphenylbiphenyl diamine $(\alpha$-NPD, $20 \mathrm{~nm})$ as the hole-transport layer, and tris-(8-hydroxyquinoline) aluminum $\left(\mathrm{Alq}_{3}, 60 \mathrm{~nm}\right)$ as the electron-transport and emitting layer. ${ }^{7-9}$ The cathode is composed of multiple functional layers to achieve both desired electrical and optical properties. Ultrathin layers of $\operatorname{LiF}(0.5 \mathrm{~nm})$ and $\mathrm{Al}(1 \mathrm{~nm})$ are deposited in sequence as the electron-injecting contact. ${ }^{3,7,8}$ A thin layer $(20 \mathrm{~nm})$ of $\mathrm{Ag}$, which has relatively low optical absorption (a)

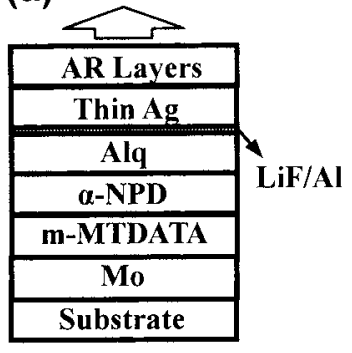

(b)

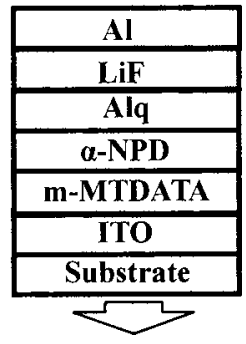

FIG. 1. Device structures of (a) the low-reflection top-emitting device, and (b) the conventional bottom-emitting device. 


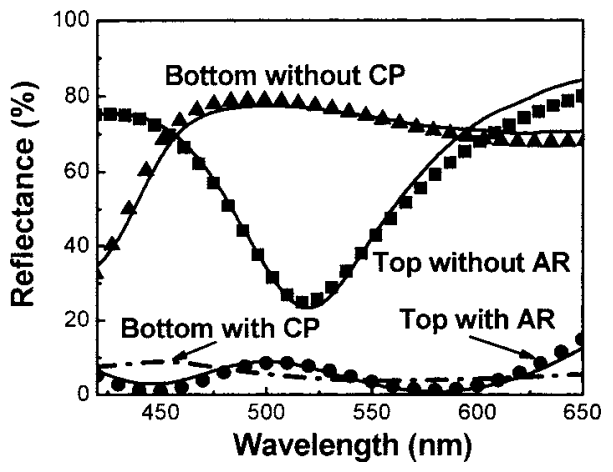

FIG. 2. Calculated (symbols) and measured (lines) reflectance spectra of the top-emitting OLEDs with and without AR coatings, and the conventional bottom-emitting OLEDs with and without lamination of the circular polarizer (CP) film.

and the highest conductivity among all metals, ${ }^{7-9}$ then overlays the $\mathrm{LiF} / \mathrm{Al}$ contact for reducing sheet resistance and for serving as the semitransparent mirror in the device structure. A 20-nm-thick Ag film gives a sheet resistance of $\sim 1 \Omega / \square$, which is one order lower than that of thick (hundreds of $\mathrm{nm}$ ) indium tin oxide (ITO). The metalorganic-metal structure is further capped with one high-index dielectric layer $\left(\mathrm{TeO}_{2}\right.$, $25 \mathrm{~nm}, n \sim 2.4-2.6$ over visible wavelengths) and one lowindex layer ( $\mathrm{LiF}, 20 \mathrm{~nm}, n \sim 1.3-1.4$ over visible wavelengths) as the anti-reflection (AR) coatings to suppress reflection from the overall device structure. In general, either dielectric, semiconductive, or conductive materials with large enough index difference could all be used in the AR layers, and these AR layers could also form part of the passivation films or the top electrode for top-emitting OLEDs. Thicknesses of the organic layers and the dielectric layers are adjusted in considering emission colors, emission efficiency, and ambient-light reflection of devices. For comparison, a conventional bottom-emitting device [Fig. 1(b)] with the same organic multilayer structure sandwiched between the ITO anode and the LiF/Al cathode was also fabricated. All material layers in devices were deposited by thermal evaporation in a multiple-source vacuum chamber without breaking vacuum.

Figure 2 shows the measured (lines) and calculated (symbols) reflectance spectra of different OLEDs: topemitting OLEDs with and without the AR coatings, and the conventional bottom-emitting OLED. For comparison, the measured reflectance spectrum of the bottom-emitting OLED laminated with a circular polarizer film is also shown. The measured spectra are in reasonable agreement with calculated ones. The reflectance of the conventional bottomemitting device is high $(\sim 80 \%)$ over most of the visible wavelengths. The top-emitting device without AR coatings exhibits reduced reflection over a limited spectral range (with a reflection minimum around $520 \mathrm{~nm}$ ), characteristic of a metal-dielectric-metal Fabry-Pérot interferometer. ${ }^{10,11}$ The location of the reflection minimum reflects the resonance wavelength of the microcavity, which was preset (mainly with the thickness of organic layers) to match the emission wavelength of the emitter $\left(\mathrm{Alq}_{3}\right)$ inside the microcavity. Further addition of the AR coatings produces dramatically suppressed reflectance over a wide band. In the structure with AR coatings, most pronounced reflections are from the bottom Mo mirror, the thin Ag mirror and the surface of highindex $\mathrm{TeO}_{2}$. By carefully adjusting phases of these three reDownloaded 17 Feb 2009 to 140.112.113.225. Redistribution subje (a) Top

(b) Bottom

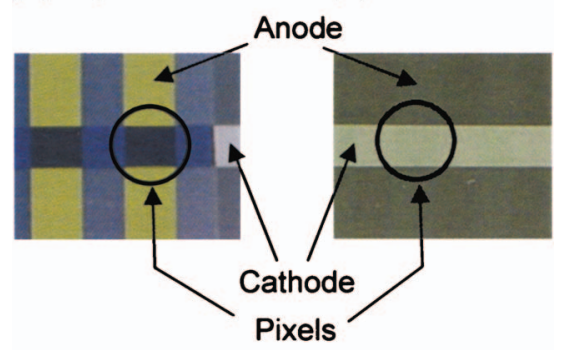

FIG. 3. (Color) Photos of (a) the low-reflection top-emitting and (b) the conventional bottom-emitting devices.

flections with organic/ $\mathrm{TeO}_{2}$ thicknesses, one in principle can place two reflection minima at desired wavelengths in the reflection spectrum as one does with conventional arts of multilayer AR coatings. ${ }^{10,11}$ In the present case, the organic/ $\mathrm{TeO}_{2}$ thicknesses were set to give two minima around 450 and $580 \mathrm{~nm}$ for obtaining a wide low-reflection band. The effect of the low-index LiF layer is less significant than that of $\mathrm{TeO}_{2}$, yet its addition is beneficial to further decrease reflection, particularly the peak value between two minima. Overall, one obtains low reflection $(<10 \%)$ over a wide spectral range, a reflectance minimum of $\sim 1 \%$ near the wavelengths to which human eyes are most sensitive, and an average reflectance of $5 \%$. The effective reflectance to human eyes under a light source with a spectral power distribution $S(\lambda)$, the luminous reflectance $R_{L}$, is defined as $R_{L}$ $=\int V(\lambda) S(\lambda) R(\lambda) d \lambda / \int V(\lambda) S(\lambda) d \lambda . V(\lambda)$ is the standard photopic curve and $R(\lambda)$ is the reflectance spectrum. Here using $S(\lambda)$ of the standard illuminating source D65, one obtains a luminous reflectance of $\sim 3.9 \%$ for the top-emitting device with AR coatings, which is over one order lower than that of the bottom-emitting device. Such a value is also slightly lower than that obtained from the conventional bottomemitting OLED laminated with a circular polarizer $\left(R_{L}\right.$ $\sim 4.5 \%$, Fig. 2). Photos in Fig. 3 show the appearance of a low-reflection top-emitting OLED and a highly reflective bottom-emitting OLED under strong illumination, clearly indicating the dark background of the present top-emitting OLED.

The inset of Fig. 4 shows the electroluminescence (EL) spectrum of the low-reflection top-emitting device, which is narrower than that of the conventional bottom-emitting device due to microcavity effects. ${ }^{7-9}$ Figure 4 compares the current-voltage-brightness characteristics of the lowreflection top-emitting device and the conventional bottomemitting device. Similar electrical characteristics of two devices [Fig. 4(a)] indicate the effectiveness of both the bottom anode and the top cathode of the top-emitting device. The top-emitting device exhibits an efficiency of $\sim 3 \mathrm{~cd} / \mathrm{A}$ [Fig. 4(b)], which is about $60 \%$ of that of the bottom-emitting device $(4.7 \mathrm{~cd} / \mathrm{A})$. Such efficiency performance represents a $\sim 1.5$ times enhancement compared to that obtained with the bottom-emitting OLED/polarizer combination, since the circular polarizer has a measured transmittance of only $\sim 40 \%$. It is also superior to those of previously reported highcontrast OLEDs. ${ }^{3-6}$ Such efficiency enhancement is attributed to the microcavity effect inherent in the present device structure. ${ }^{7-9}$ Even higher efficiency enhancement could be obtained using bottom electrodes of higher reflectivities (e.g., $\mathrm{Ag}$ and $\mathrm{Al}$, etc.), but the low-reflection band of the to AlP license or copyright; see http://apl.aip.org/apl/copyright.jsp 
(a)
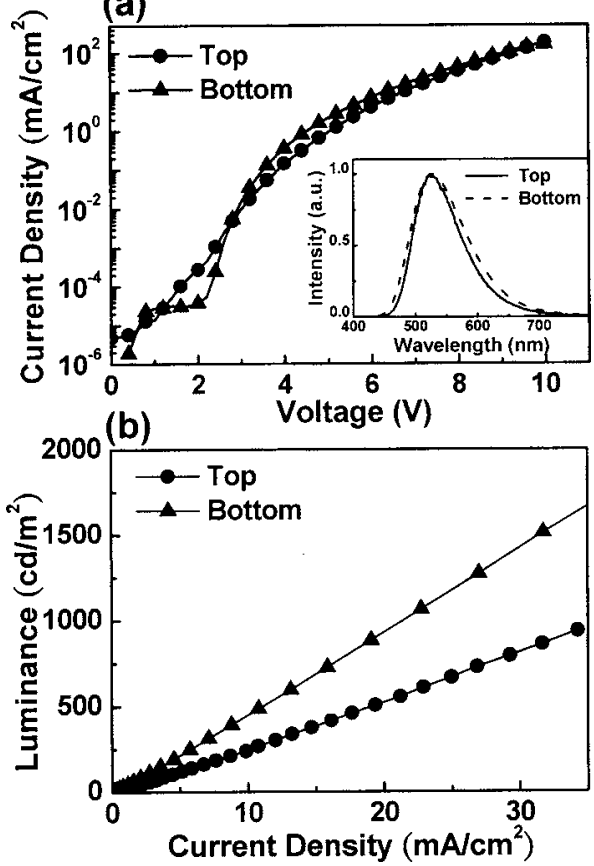

FIG. 4. (a) $I-V$ characteristics and (b) $L-I$ characteristics of the lowreflection top-emitting OLED and the conventional bottom-emitting OLED. Inset: EL spectra of the two devices.

metalorganic-metal interferometer would become substantially narrower, resulting in overall increase of reflection from the whole structure. Thus there is trade-off between the efficiency and the contrast.

The present low-reflection, top-emitting OLEDs are readily compatible with the processing of active-matrix backplanes and have been successfully implemented into a 3.8 in. (1/4 video graphic array, i.e., with $240 \times 320$ pixels) AMOLED using the typical two-transistor-one-capacitor pixel circuit. ${ }^{12}$ The cross section of the AMOLED is shown in Fig. 5(a), which shows the integration of high-contrast top-emitting OLEDs and top-gate low-temperature polycrystalline silicon thin-film transistors (TFTs). The fabrication of the active matrix adopts self-aligned $p$-channel TFT processing, seven mask sets and three metal processes. ${ }^{12}$ Figure 5(b) shows the side-by-side comparison of displayed images from the present low-reflection top-emitting AMOLED (right) and a conventional bottom-emitting AMOLED (left) under a strong lighting environment $(\sim 1000$ lux $)$. One sees that while the low-reflection top-emitting AMOLED still exhibits an image of clear contrast, the conventional bottom-emitting AMOLED shows strong reflection and image washout.

In summary, we report a high-contrast OLED structure that utilizes only optical characteristics of electrodes and anti-reflection coatings deposited outside the active region of devices, thus reducing the complexity of devices. Such a structure is implemented into top-emitting OLEDs, and the microcavity structure inherent with devices gives improved EL efficiency compared to that obtained from conventional OLEDs laminated with polarizers or from previous high-

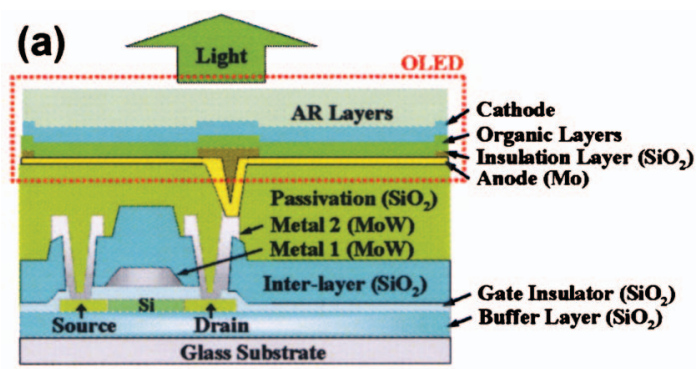

(b)

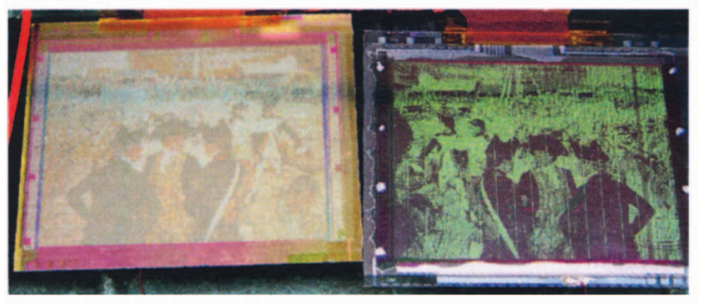

FIG. 5. (Color) (a) Cross section of the top-emitting AMOLED; (b) photos of the low-reflection top-emitting (right) and the conventional bottomemitting (left) AMOLEDs.

contrast OLEDs. AMOLEDs incorporating such highcontrast top-emitting OLEDs and thus having improved readability under a strong lighting environment are also demonstrated. Although only top-emitting devices are discussed in this letter, such a scheme is applicable to bottom-emitting devices as well if one inverts the structure, i.e., with a reflective electrode on top and semitransparent electrode/AR coatings at the bottom.

The authors would like to acknowledge financial support from the National Science Council and ITRI of the Republic of China. One of the authors (C.L.L.) is also grateful for financial support from a MediaTek Fellowship. The authors also thank Dr. Bennet Chu of RiTdisplay Corporation for providing circular polarizer films for comparative studies.

${ }^{1}$ C. W. Tang and S. A. VanSlyke, Appl. Phys. Lett. 51, 913 (1987).

${ }^{2}$ M.-H. Lu, M. S. Weaver, T. X. Zhou, M. Rothman, R. C. Kwong, M. Hack, and J. J. Brown, Appl. Phys. Lett. 81, 3921 (2002).

${ }^{3}$ L. S. Hung and J. Madathil, Adv. Mater. (Weinheim, Ger.) 13, 1787 (2001).

${ }^{4}$ A. N. Krasnov, Appl. Phys. Lett. 80, 3853 (2002).

${ }^{5}$ H. Aziz, Y.-F. Liew, H. M. Grandin, and Z. D. Popovic, Appl. Phys. Lett. 83, 186 (2003).

${ }^{6}$ S. H. Li, H. Liem, C. W. Chen, E. H. Wu, Z. Xu, and Y. Yang, Appl. Phys. Lett. 86, 143514 (2005).

${ }^{7}$ C.-W. Chen, P.-Y. Hsieh, H.-H. Chiang, C.-L. Lin, H.-M. Wu, and C.-C. Wu, Appl. Phys. Lett. 83, 5127 (2003).

${ }^{8}$ C.-C. Wu, C.-L. Lin, P.-Y. Hsieh, and H.-H. Chiang, Appl. Phys. Lett. 84, 2966 (2004).

${ }^{9}$ C.-W. Chen, C.-L. Lin, and C.-C. Wu, Appl. Phys. Lett. 85, 2469 (2004).

${ }^{10}$ M. Born and E. Wolf, Principles of Optics, 7th ed. (Cambridge University Press, Cambridge, 1999).

${ }^{11}$ O. S. Heavens, Optical Properties of Thin Solid Films (Dover, New York, 1991).

${ }^{12}$ Y.-H. Yeh, J.-R. Shih, C.-R. Chen, Y.-H. Kuo, Y.-L. Liu, C.-C. Cheng, C.-F. Chung, C.-C. Chen, C.-Y. Huang, H.-Y. Tseng, T.-H. Chen, and M.-D. Chen, Proceedings of Tenth International Display Workshops (IDW), Fukuoka, Japan, 2003, p. 259. 